\title{
Characteristic Comparison of U-Shaped Monopole and Complete Monopole Antenna.
}

\author{
Zahid Gulzar Khaki ${ }^{1}$, Showkat Ahmad Dar ${ }^{2}$ \\ ${ }^{1}$ (Department of Electronics \& communication Engineering, Islamic University of Science and Technology, India) \\ ${ }_{2}^{2}$ (Department of Computer Science Engineering, Islamic University of Science and Technology, India)
}

\begin{abstract}
A monopole antenna is a type of radio antenna formed by replacing one half of a dipole antenna with a ground plane at right-angles to the remaining half. Monopoles may be used from a few hundred KHz through several GHz in frequency and are commonly one-quarter of a wave length long, but may be shorter or longer. Monopole antennas exhibit high gain and improved efficiency in a surprisingly small package. Monopole antenna can be designed to exhibit wideband capabilities. The different available monopole antennas are dual band printed monopole antenna, cross-slot monopole antenna, U-shaped monopole antenna, triangular shaped monopole antenna and a wideband monopole antenna. This paper deals with the comparison obtained from the results such as return loss, VSWR, current distribution, and the radiation pattern of simple U-shaped and complete monopole antenna.
\end{abstract}

Keywords- CPW, CST, FR4, LAN, WIMAX

\section{Introduction}

An antenna (or aerial) is a transducer designed to transmit or receive electromagnetic waves. In other words, antennas convert electromagnetic waves into electrical currents and vice versa. They are used with waves in the radio part of the electromagnetic spectrum, that is, radio waves, and are a necessary part of all radio equipment. In air, those signals travel very quickly and with a very low transmission loss. The signals are absorbed when moving through more conductive materials, such as concrete walls or rock. Antennas are used in systems such as radio and television broadcasting, point-to-point radio communication, wireless LAN, cell phones, radar, and spacecraft communication. Antennas are most commonly employed in air or outer space, but can also be operated under water or even through soil and rock at certain frequencies for shorter distances. There are two fundamental types of antenna directional patterns, which, with reference to a specific two dimensional plane are usually horizontal (parallel to the ground) or vertical (perpendicular to the ground).

\subsection{Monopole antenna}

A monopole antenna is a type of radio antenna formed by replacing one half of a dipole antenna with a ground plane at right-angles to the remaining half. If the ground plane is large enough, the monopole behaves like a dipole, as if its reflection in the ground plane formed the missing half of the dipole, except that the radiation from the reflected half is added to that of the real half [1]. However, a monopole will have a directive gain of 5.19dB (gain is twice (3dB over) that for a half-wave dipole antenna), and a lower input resistance. Monopoles may be used from a few hundred $\mathrm{KHz}$ (navigation beacons and broadcast stations) through several $\mathrm{GHz}$ in frequency and are commonly one-quarter of a wave length long, but may be shorter or longer.

The impedance of a monopole is one half that of a full dipole antenna. For a quarter-wave monopole $(\mathrm{L}=0.25 * \lambda)$, the impedance is half of that of a half-wave dipole, so $\mathrm{Z}_{\mathrm{in}}=36.5+\mathrm{j} 21.25 \mathrm{ohms}$. The directivity of a monopole antenna is directly related to that of a dipole antenna. If the directivity of a dipole of length $2 L$ has a directivity of $\mathrm{D}_{1}$ [decibels], then the directivity of a monopole antenna of length $L$ will have a directivity of $\mathrm{D}_{1}+3$ [decibels]. That is, the directivity (in linear units) of a monopole is twice the directivity of a dipole antenna of twice the length. The reason for this is simply because no radiation occurs below the ground plane; hence, the antenna is effectively twice as "directive".

\section{Design Of Monopole Antenna}

The size of the antenna on the substrate should be very small. Conventional whip and helical antennas takes large volume on the substrate. So in order to reduce the size of the antenna on the substrate, we use Micro strip antennas. The objective of designing the Monopole antenna is the miniaturization of antenna. Monopole antennas exhibit high gain and improve efficiency in a surprisingly small package. Monopole antenna can be designed to exhibit wideband capabilities. 


\subsection{Selection of Antenna specifications}

The selection of antenna parameters is influenced by its practical applications. The antenna specifications are chosen such that it meets all our requirements. The four antenna specifications to be chosen are frequency of operation, feed technique, dielectric constant and thickness of dielectric substrate.

\subsubsection{Frequency of Operation}

Bluetooth /ISM, WiMAX, WLAN takes the advantage of a license free frequency bands, i.e. Industrial Scientific and Medical (ISM) bands. The ISM bands for long range communication have center frequency of $2.5 \mathrm{GHz}, 3.5 \mathrm{GHz}$ and $5.5 \mathrm{GHz}$.

\subsubsection{Feed Technique}

Here, we have chosen $50 \Omega$ coplanar waveguide (CPW) feed line method as our feeding technique as the matching is easier and the process is simpler.

\subsubsection{Dielectric Constant}

The substrate is chosen such that the antenna has a low Q-factor, as the Q-factor is inversely proportional to bandwidth [2]. Another factor taken into consideration when selecting a substrate is loss tangent $(\tan \delta)$. The dielectric loss tangent of FR4 substrate is 0.0245. Lower the loss tangent higher the bandwidth so a dielectric with low $\tan \delta$ value is preferred in order to have larger bandwidth.

\subsubsection{Thickness of Substrate}

There are numerous substrates that can be used for the design of micro strip antennas, and their dielectric constants are usually in the range of $2.2 \leq \boldsymbol{E}_{r} \leq 12$. The one that are most desirable for antenna performance are thick substrates whose dielectric constant is in the lower end of the range because they provide efficiency, larger bandwidth, loosely bound fields for radiation into space, but at the expense of larger element size. Thin substrates with higher dielectric constants are desirable for microwave circuitry because they require tightly bound fields to ever, because of their greater losses; they are less efficient and have relatively smaller bandwidths. Since microstrip antennas are often integrated with other microwave circuitry, a compromise has to be reached between good antenna performance and circuit design. Bandwidth is directly proportional to the thickness of the substrate. The thickness chosen is $1.6 \mathrm{~mm}$.

\subsubsection{Design of simple $U$-shaped monopole antenna}

For the simple U-shaped antenna, it is seen that a first resonant mode at about $2.5 \mathrm{GHz}$ and a second mode at about $5.2 \mathrm{GHz}$ are obtained for the U-shaped monopole only. For the proposed antenna, the first resonant mode of the U-shaped monopole at about $2.5 \mathrm{GHz}$ is successfully excited with good impedance matching to cover the $2.4 \mathrm{GHz}$ WLAN and $2.5 \mathrm{GHz}$ Wi-MAX bands. The resonating path length of the Ushaped monopole is given as $L_{11}\left(L_{11}=S+L_{3}+L_{1}\right)$. Thus the designed U-shaped monopole antenna is simulated using CST software.

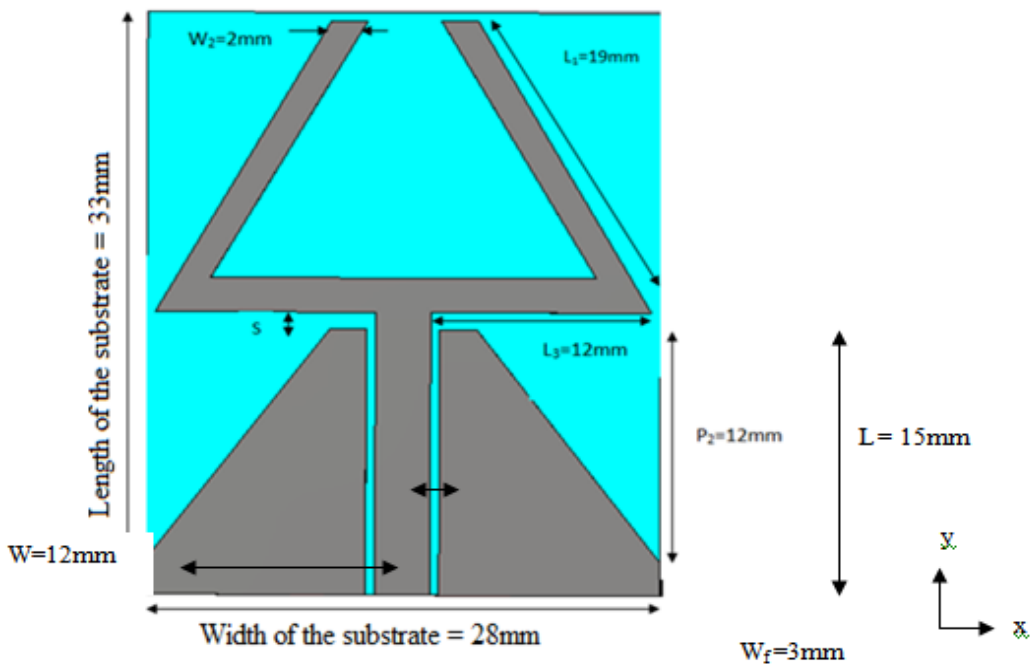

Fig.2.1: Structure of simple U-shaped monopole antenna 


\section{Results Of Different Structures Of Monopole Antenna}

\subsection{Results Obtained For U-Shape Monopole Antenna}

\subsubsection{Return loss Vs Frequency}

When the feed is given to the U-Shaped Monopole antenna, reflection occurs at the port because of impedance mismatching. It means that when a load is mismatched, not all of the available power from the generator is delivered to the load. This loss is termed as return loss. But, when the feed is perfectly matched return loss will be minimum. It is given using the formulae

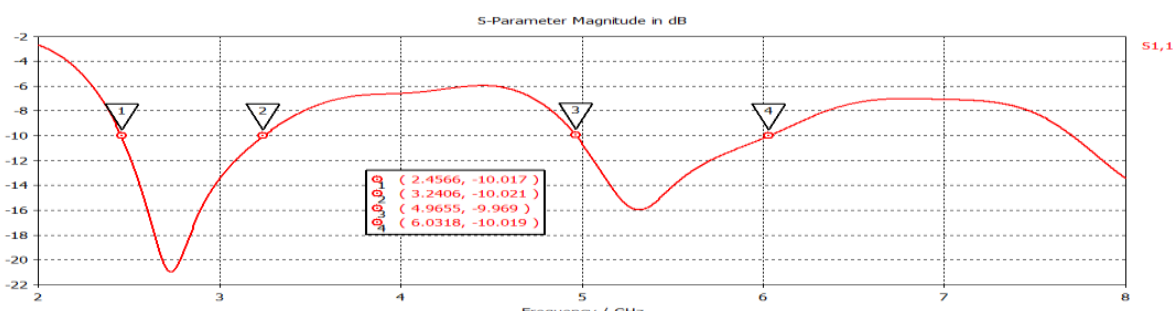

Fig 3.1: Return loss Vs Frequency plot

\subsubsection{VSWR Vs Frequency}

From the graph it is observed that VSWR for Monopole antenna is below 2 at a both frequencies

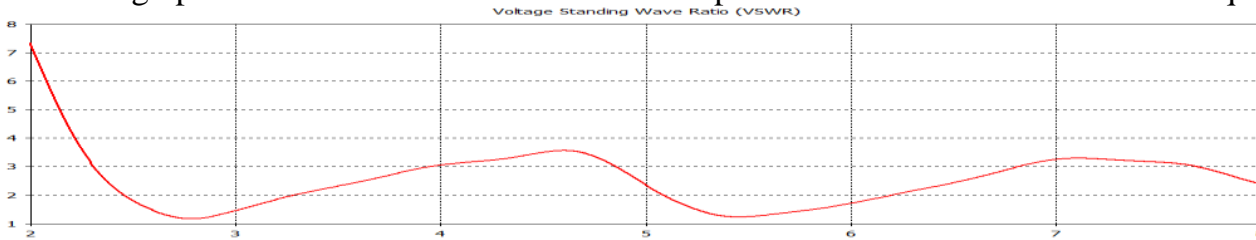

Fig 3.2. VSWR Vs Frequency plot

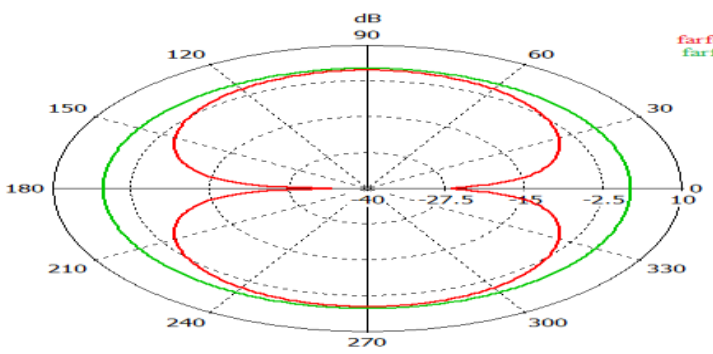

Fig 3.3 2D Radiation patern at $2.7 \mathrm{GHz}$

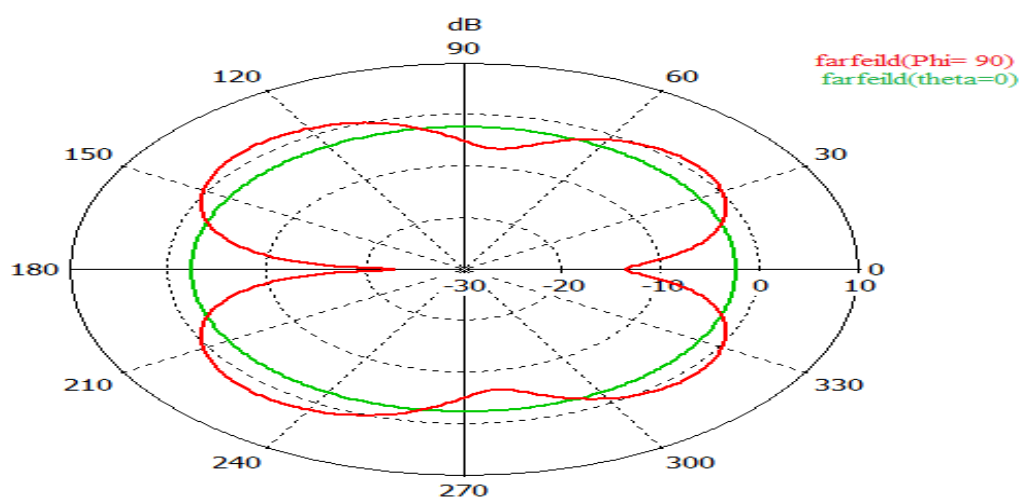

Fig 3.4. 2D Radiation patern at $5.4 \mathrm{GHz}$ 


\subsection{Results obtained for Monopole Antenna}

3.2.1 Return loss Vs Frequency

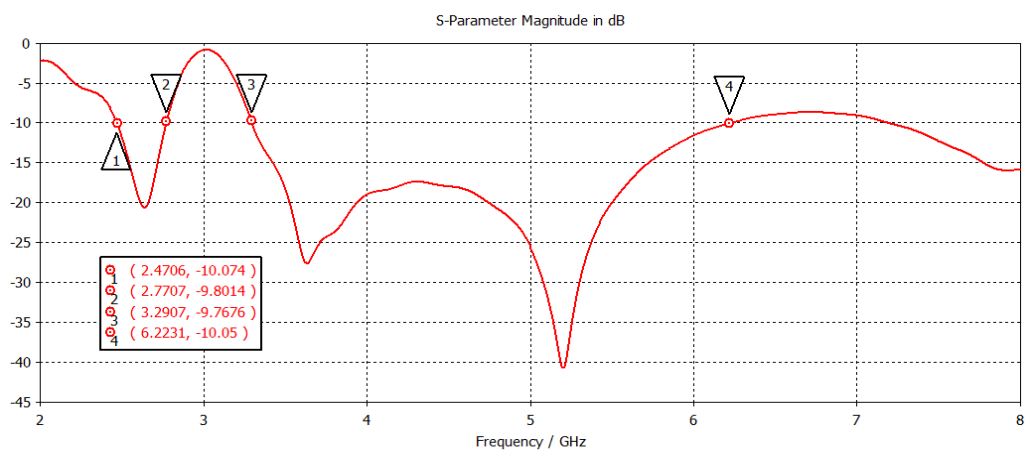

Fig 3.5 Return loss Vs Frequency plot

\subsubsection{VSWR Vs Frequency}

From the graph it is observed that VSWR for Monopole antenna is below 2 at a resonating frquency

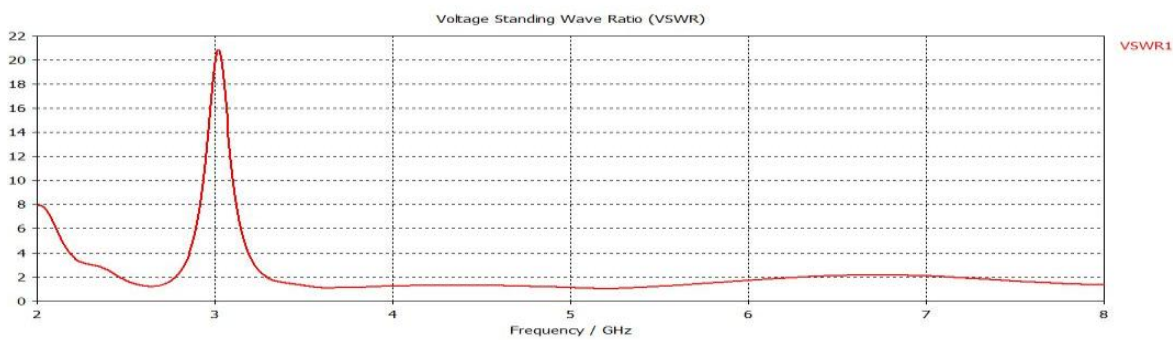

Fig 3.6: VSWR Vs Frequency plot

\subsubsection{Current Distribution at $2.6 \mathrm{GHz}$ and $5.2 \mathrm{GHz}$}

At the $2.6 \mathrm{GHz}$ frequency the U-shaped monopole sleeves exhibits maximum current which is shown in the Fig 3.7. The sleeves of the monopole antenna cause the first resonance frequency at the lower band.
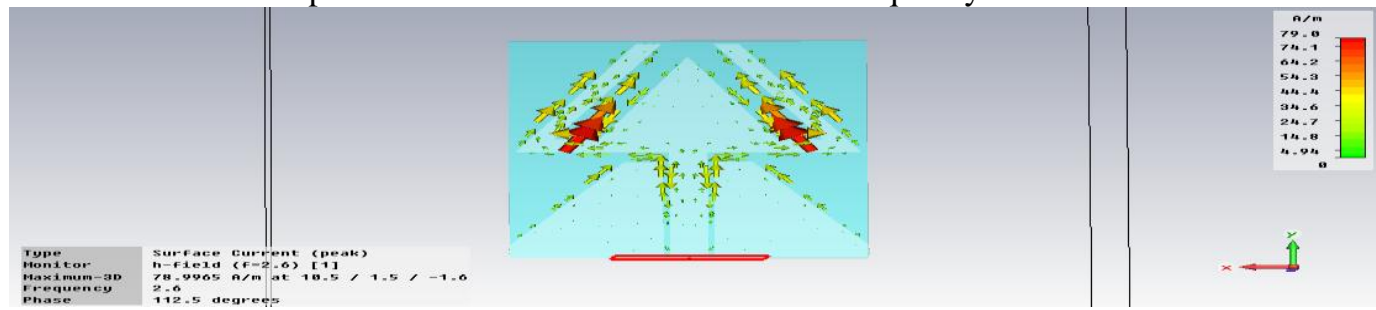

Fig 3.7.Current distribution at $2.6 \mathrm{GHz}$

At the $5.2 \mathrm{GHz}$ strong surface currents are seen around the bottom of the monopole antenna. This is due to the electromagnetic coupling between U-shaped and Triamgular monopole. The result indicate that the U-shaped monopole and the Triangular monopole are the major radiating elements for the proposed antenna at higher band.

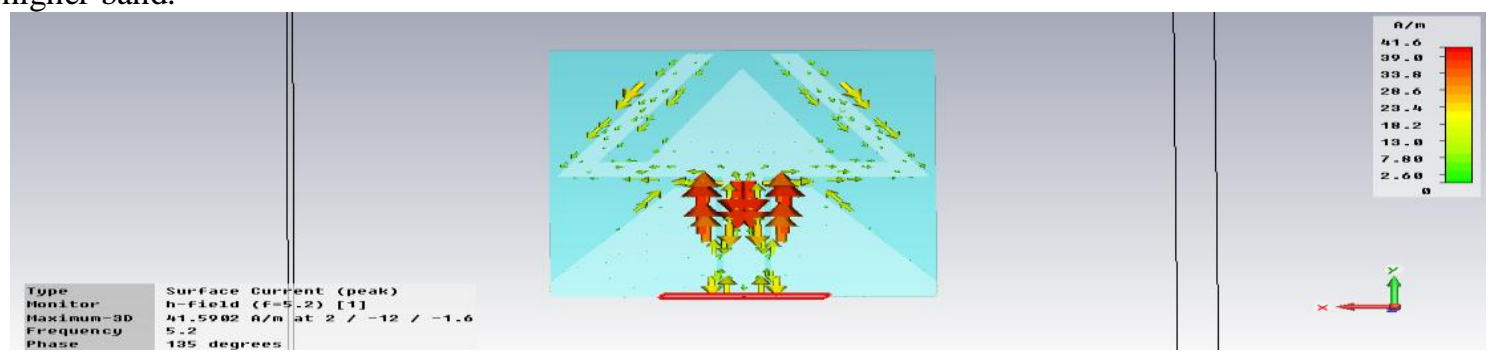

Fig 3.8 :Current distribution at $5.2 \mathrm{GHz}$ 


\subsubsection{D Radiation pattern at $2.6 \mathrm{GHz}$ and $5.2 \mathrm{GHz}$}

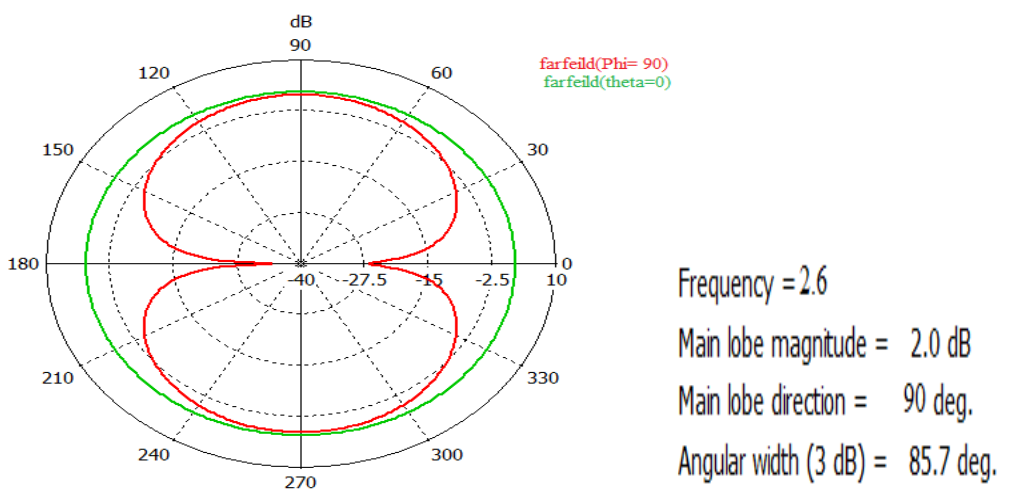

Fig 3.9 2D Radiation patern at $2.6 \mathrm{GHz}$

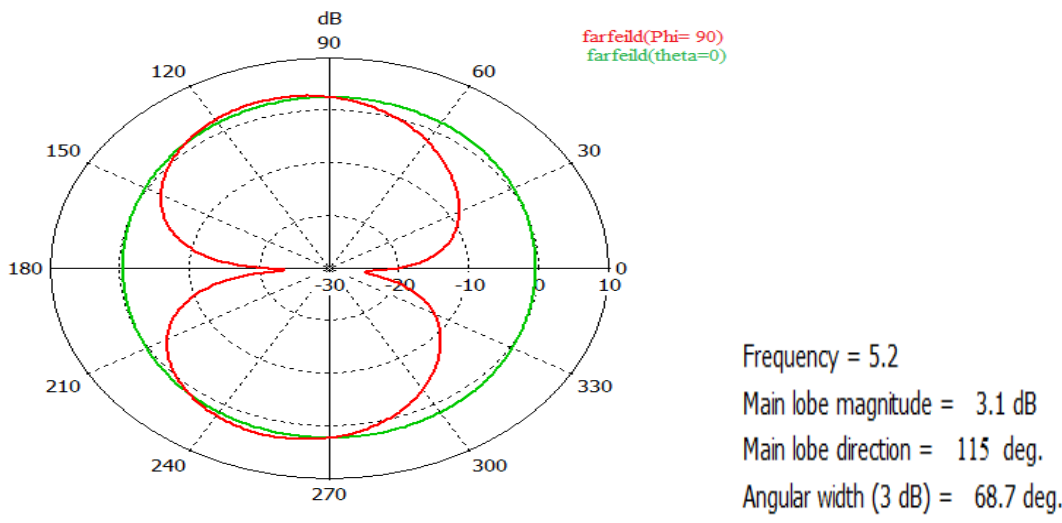

Fig 3.10. Radiation patern at $5.2 \mathrm{GHz}$

\section{Conclusion}

The U-shaped monopole has a good return loss at the two bands, although the first resonant mode is shifted to the higher frequency. The surface current of the monopole antenna shows the current distribution at the respective frequency of operation. From the radiation pattern, one can infer the figure of eight pattern in the E-plane and the Omni-directional pattern in the H-plane. The antenna shows the good dipole like radiations characteristics with the moderate gain over the operating bands which are attractive for practical applications in the communication gadgets. A good agreement between the simulated and measured results is obtained.

\section{References}

[1] Constantine A. Ballanis “Antenna theory - analysis and design” second edition, John wiley \& son's .inc.

[2] L. Setian, "Practical Communication Antennas with Wireless Applications", Prentice Hall PTR, New Jersey: 1998.

[3] E.D. Caswell, W.A. Davis, and W.L. Stutzman, "Fundamental Limits on Antenna Size," Artech House, Boston: 1992.

[4] Girish Kumar and K P Ray "Broadband micro strip antennas" by, Artech House antennas and propagation library.

[5] C. Y. Pan, T. S. Horng, W. S. Chen, and C. H. Huang, "Dual wideband printed monopole antenna for WLAN/WiMAX applications," IEEE Antennas Wireless Propag. Lett., vol. 6, pp.149-151, 2007.

[6] Design of Dual-band CPW-fed cross-slot monopole antenna for WLAN operation" was presented in IEEE Antennas Wireless Propag. Lett.,, vol,. 6, 2008.

[7] Qing-Xin Chu and Liang-Hua Ye "A compact dual-frequency antenna with triangular ring monopole and U-shaped sleeves", European microwave conference, 2009.

[8] Y.T. Lo, D. Solomon, "Theory and Experiment on Microstrip Antennas," IEEE Trans. Ant. Prop., vol. AP-27, no. 2, March 1989, pp. 137-145.

[9] Qing-Xin Chu and Liang-Hua Ye "Desig dual-wideband antenna for WLAN/WIMAX applications ," IEEE Trans.Antenna Propag., VOL. 58, NO. 12, Dec 2010. 